\title{
Lateral meniscal posterior root tears experience acceptable healing status after transtibial repair technique
}

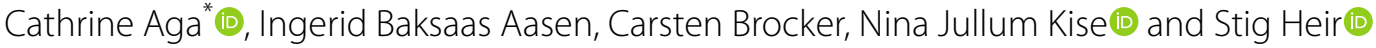

\begin{abstract}
Purpose: To evaluate patient MRI results, demography and clinical outcome following transtibial repair of lateral and medial meniscal posterior root tears.

Methods: Patients treated with transtibial repairs of posterior meniscal root tears from 2015 through 2018 performed pre- and postoperative MRI scans. Outcome measures were continuity/discontinuity of the meniscal root and change in meniscal extrusion on MRI. Other outcomes were KOOS, Lysholm score, Tegner activity scale and the Global Rate of Change (GRoC) score for function and pain at follow-up.
\end{abstract}

Study design: Retrospective case-series.

Results: Of 41 patients, 36 attended follow-up at mean 26 (12-38) months postoperatively. At follow-up, 11 out of 18 lateral meniscus posterior root tear (LMPRT) versus 5 out of 18 medial meniscus posterior root tear (MMPRT) repairs were classified as healed. Meniscal extrusion decreased in LMPRTs from of $2.3 \pm 1.5 \mathrm{~mm}$ to $1.4 \pm 1.09 \mathrm{~mm}(p=0.080)$ and increased in MMPRTs from $3.1 \pm 1.6 \mathrm{~mm}$ to $4.8 \pm 1.9 \mathrm{~mm}(p=0.005)$ at FU (between-group difference, $p<0.001$ ). LMPRT repairs were associated with $\mathrm{ACL}$ injury and additional meniscal injury and were younger and with lower BMI. No between-group differences were found for KOOS, Lysholm or GRoC Function scores. Tegner scale was higher and GRoC Pain score lower in the LMPRT group compared to the MMPRTs.

Conclusion: Following transtibial repair for meniscal posterior root repairs, the LMPRTs had a higher frequency of healing, whereas most MMPRTs continued to extrude, despite surgical intervention. The study confirmed that LMPRTs and MMPRTs differ in demography and associated injuries.

\section{Background}

Posterior root tears of the medial and lateral meniscus are known to have devastating consequences for the knee if left untreated [1, 4, 9, 32]. Currently, the transtibial meniscal root repair is the surgical treatment of choice and with this technique the intraarticular contact pressures and tibiofemoral contact areas can be restored $[8,10,23,30]$. Although the surgical treatment options are identical for both medial and lateral meniscal posterior root tears, these two conditions arise in different

*Correspondence: cathrineaga@gmail.com

Orthopaedic Department, Martina Hansens Hospital, Baerum, Norway populations and are associated with different underlying factors $[13,20]$. Clinical studies have shown acceptable outcomes after repair for both conditions [5, 17, 24].

MRI scans of the affected knees can be used to evaluate the degree of healing of the posterior root repairs $[12,31]$. Both continuity of the meniscal root at footprint and the meniscal extrusion out of the tibiofemoral joint are correlated with the prognosis and patient related outcome after repair $[8,9,27]$. Whether medial and lateral posterior meniscal root repair show a difference in their aspects of healing, is of interest when considering treatment options for these kinds of injuries. There are relatively few studies published on the outcome after 
transtibial root repair of medial and lateral menisci, and no study compare MRI results between the two conditions [20].

The primary purpose of this study was to verify the degree of healing and the degree of meniscal extrusion in the two groups LMPRTs and MMPRTs after transtibial root repair. Secondary purposes were to look at demography and clinical outcomes of the same two groups. Our hypothesis was that there would be a difference in the potential for healing and change in meniscal extrusion after repair of LMPRT compared to repair of MMPRT as evaluated by MRI at a minimum of 1 year follow-up.

\section{Study design}

Retrospective case-series, Level IV.

\section{Material and methods}

The study was performed at a single hospital specialized in orthopedic surgery. The hospital administrative system was used to retrospectively identify patients treated with transtibial repair of medial or lateral meniscal root tears. This treatment was first implemented at the hospital in 2015 and all patients exposed to this procedure during April 2015 through June 2018 were invited to attend to the study. Patients that were not able to understand and speak Norwegian language or not able/willing to meet to the hospital for a clinical examination were excluded from the study. Patients having additional ligament injury of the MCL, PCL, PLC or LCL or simultaneous alignment corrections (osteotomy) procedures, were also not included in the study. Patients with Kellgren-Lawrence (KL) grade three or four at preoperative radiographs or ICRS score three or four at surgery were not considered for root repair.

All study participants received information about the study and signed a consent. Information and details from the surgical procedures including concurrent intraarticular pathology were extracted from the patients' journals. Follow-up clinical examination were performed by two experienced orthopedic surgeons together with a physiotherapist. All patients were referred to their nearest radiographic institute to perform a new MRI scan at follow-up. Pre- and postoperative MRI scans were examined and evaluated by a radiologist specialized in musculoskeletal radiology. The methodology of evaluating the MRIs were discussed between the authors based upon a literature search $[18,28,31]$. Location, appearance and continuity/discontinuity of the fibers and the degree of extrusion were recorded [25]. To ensure the reliability of the findings, the inter-rater reliability (ICC) between three observers was measured.

\section{Surgery}

Anteromedial and anterolateral portals were established, a standard arthroscopy of the joint was performed and the posterior roots of the menisci were evaluated. If a posterior meniscal root tear was present, the transtibial fixation technique was performed in a standardized approach [3, 7, 22]: Additional portals and cannulas were established if necessary. The footprint location for root attachment was prepared with curette and shaver. An anatomic footprint was aimed for [14]. The root was loosened from adherent scar tissue if necessary, and the meniscofemoral ligament preserved if present. Sutures were thread through the meniscal root with a suture machine and although two sutures were aimed for, different suture techniques and materials were used. An external aiming drill-guide was used to drill a tunnel from anteromedial or anterolateral tibia towards the desired fixation site at the posterior tibial plateau [21,33]. Either retrograde or anterograde overdrilling of the guide was performed to establish the transtibial tunnel. The sutures were shuttled through the tunnel with a nitinol passing wire and then the meniscal root was reduced down towards the footprint through suture tensioning. Fixation was performed on the anteromedial or anterolateral tibia by tying the suture ends over a suture button (TightRope $\mathrm{ABS}^{\circledR}$, Arthrex, Naples, US). The postoperative rehabilitation program consisted of non-weightbearing on crutches without brace-support for 6 weeks postoperatively, partial weight bearing for another 6 weeks and then gradually increased weightbearing until 4 months postoperatively. Knee bending of more than 90 degrees with load was prohibited until 3-4months postoperatively [30].

\section{Outcomes}

Demographic and surgical data was extracted from the patients journals: Age, gender, smoking habits, left or right knee, medial or lateral injury, concomitant injuries (ACL injury or other meniscal or cartilage injuries) and additional surgical procedures (ACL primary reconstruction or ACL revision procedures, additional meniscal or cartilage surgery) were recorded. Any reoperations of the affected knees were recorded.

\section{MRI}

The continuity/discontinuity of the menisci in all three planes (axial, coronal and sagittal) and amount of extrusion $(\mathrm{mm})$ were used to evaluate healing after repair. An intact repair was only characterized by root continuity in all three planes, a partial repair was characterized by partial discontinuity in one or two planes (coronal, sagittal or axial plane), and an incomplete repair was characterized 


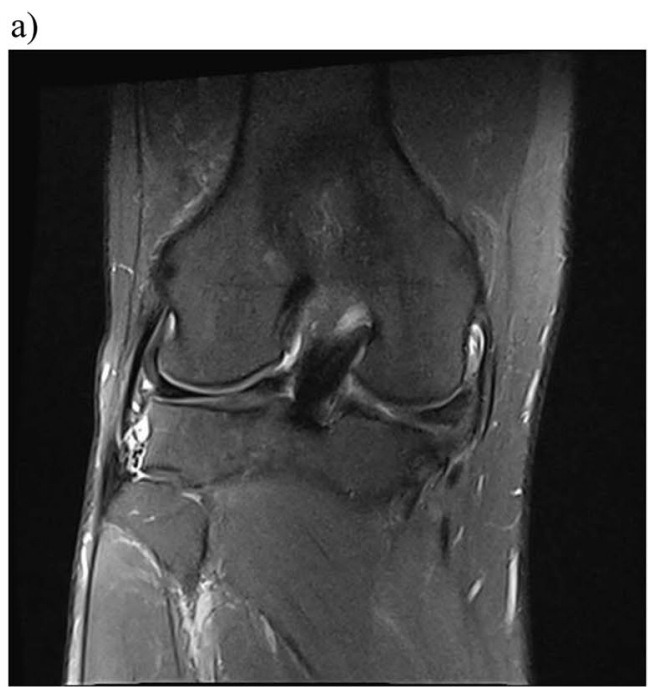

b)

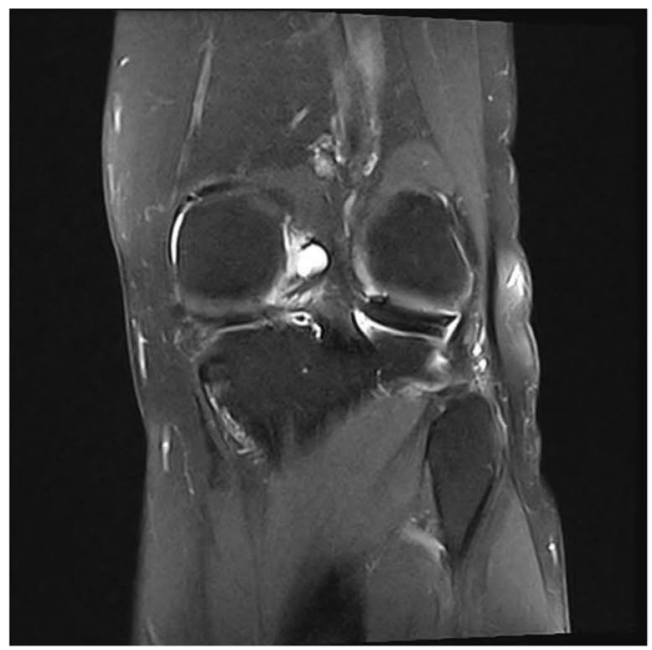

c)

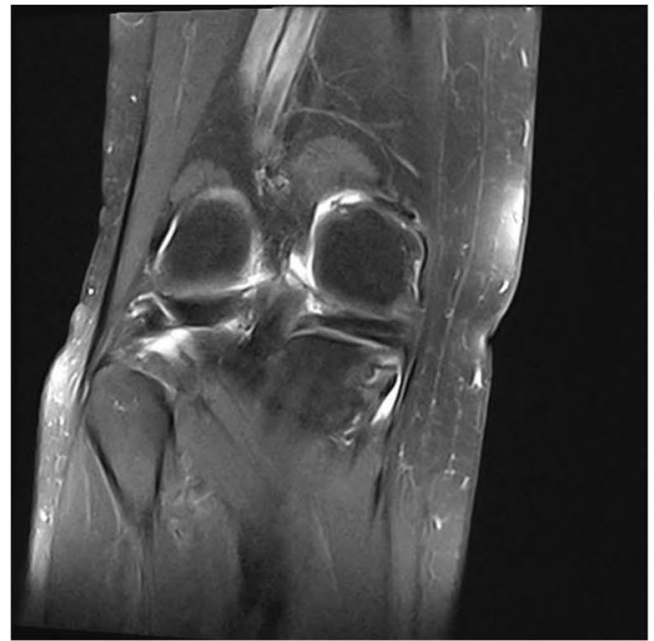

Fig. 1 The figures present a (a) non-healed, (b) partially healed and (c) a healed medial meniscus posterior root tear (MMPRT) repair by complete discontinuity (Fig. 1) [18]. The meniscal extrusion pre- and postoperatively was measured at the broadest level of the eminentia medialis and lateralis in the coronal plane and reported in $\mathrm{mm}$ [25].

\section{Radiographic imaging}

Anteroposterior radiographs of the knees were used to evaluate the degree of radiographic osteoarthritis according to the Kellgren-Lawrence (K-L) classification [16].

\section{Clinical outcomes}

Each patient filled out patient reported outcome measures (PROMs) including the Knee Injury and Osteoarthritis Outcome Score (KOOS), Tegner activity scale and Lysholm score [34, 35]. The Global Rating of Change (GRoC) score was presented as a Likert scale from 0 to 5 $(0=$ very much better and $5=$ very much worse). GRoC was measured for both function (GRoC Function) and pain (GRoC Pain) [15].

\section{Statistics}

Demographic data were presented in counts and percentages for nominal variables and with means and ranges for the continuous variables. A paired t-test was used to compare the change of extrusion within each group, ordinal data was compared by the independent $t$-test or Mann-Whitney test. For comparison of between-group differences of categorical data a chi-square test or Fisher exact test was used. Significance level was set at $5 \%$ $(p=0.05)$. To assess measurement reliability, the single measures, absolute agreement definition of the intraclass correlation coefficient (ICC) for inter-rater reliability was calculated with a two-way random effects model. All analyses were performed in IBM SPSS $\odot$ statistical software Version 25. Post hoc power calculation revealed that a with 18 participants in each group and $1 \mathrm{~mm}$ difference in extrusion set as a clinical meaningful difference between the two groups, the study showed a power of $70 \%(p=0.05, \mathrm{SD}=0.9 \mathrm{~mm})$.

\section{Results}

Forty-one patients had a transtibial suture repair of their meniscal posterior root tear during the inclusion period. Thirty-six (88\%) of the patients participated in the study (Fig. 1). The mean follow-up time was mean of 26 months (range 12 to 38 months). Eighteen patients had a medial posterior root repair and 18 patients a lateral posterior root repair. Baseline demographics and clinical characteristics of both patient groups are presented in Table 1. Most patients with LMPRTs were treated for a concomitant ACL rupture and in 11 of 18 an additional meniscal injury was found. Patients with LMPRTs had surgery at younger age and presented lower BMI than patients with 
Table 1 Baseline demographics and surgical data of study patients with posterior meniscal root repairs for the medial and lateral meniscus

\begin{tabular}{llll}
\hline & Medial meniscus $(\boldsymbol{n = 1 8 )}$ & Lateral meniscus $(\mathbf{n}=\mathbf{1 8})$ & $\mathbf{P}$-values \\
\hline Age (median \pm IQ range) & $54.6 \pm 15(35-65)$ & $26.3 \pm 11(18-45)$ & $<\mathbf{0 . 0 5 *}$ \\
Sex (male/female) & $7 / 11$ & $8 / 10$ & $\mathrm{~ns} \#$ \\
Side (right/left) & $9 / 9$ & $6 / 12$ & $\mathrm{~ns} \#$ \\
Body mass index (mean \pm SD) & $29.1 \pm 4.7$ & $25.3 \pm 2.2$ & $<\mathbf{0 . 0 5 *}$ \\
ACL reconstruction(n) & 0 & 17 & $<0.05 \#$ \\
ACL revisions(n) & 0 & 6 & $<0.05 \#$ \\
Additional mensical injury(n) & 0 & 11 & $<0.05 \#$ \\
Follow-up (months) & 26 & 25 & $n s^{*}$ \\
\hline
\end{tabular}

* Students t-test/Mann Whitney test

\#Chi-square/Fishers exact test

ns Non significant

MMPRTs. Cartilage degeneration (ICRS grade 1 or 2) was detected at surgery in 5 of the 18 LMPRT compared to 17 of 18 MMPRTs. (Fig. 2)

\section{MRI}

The degree of healed, partially healed and incomplete healing of the LMPRTs and MMPRTs, classified by MRI are presented in Table 2. LMPRTs showed the highest amount of healing and the MMPRTs showed the highest amount of meniscal extrusion at follow-up. The change in meniscal extrusion measured from pre- to postoperatively are presented in Table 2. LMPRT repairs: MRI in $18 / 18$ patients showed a mean preoperative meniscal extrusion at $2.3 \pm 1.49 \mathrm{~mm}$ and a mean postoperative extrusion at $1.4 \pm 1.09 \mathrm{~mm}(p=0.08)$. In 11 of the 18 patients the rupture was classified as healed. MMPRT repairs: MRI from $18 / 18$ patients showed a change in meniscal extrusion from $3.1 \pm 1.55 \mathrm{~mm}$ preoperatively to $4.8 \pm 1.90 \mathrm{~mm}$ postoperatively $(p<0.05)$. In 5 of these 18 patients the suture was classified as healed on MRI at follow-up. There was a significant difference between groups in meniscal extrusion from pre- to postoperatively

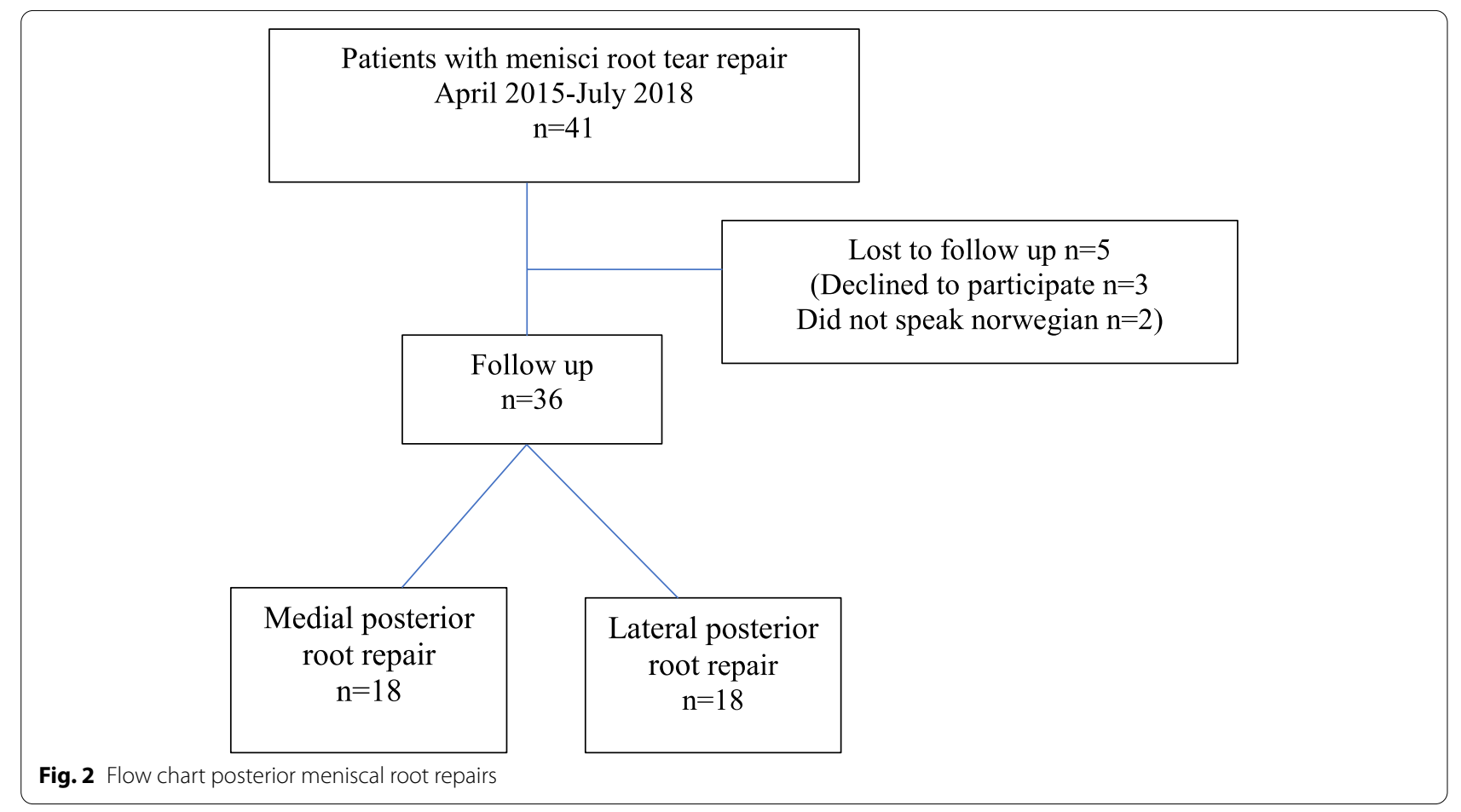


Table $2 \mathrm{MRI}$ and radiographic findings of Meniscus posterior root repairs

\begin{tabular}{|c|c|c|c|}
\hline & $\begin{array}{l}\text { Medial meniscus. Root } \\
(n=18)\end{array}$ & $\begin{array}{l}\text { Lateral meniscus root } \\
(n=18)\end{array}$ & $P$-value \\
\hline \multicolumn{4}{|l|}{ MRI: } \\
\hline Menisci healing status: complete/partial/non-healed (n) & $5 / 8 / 5$ & $11 / 5 / 2$ & ns\# \\
\hline Complete healing: & 5 & 11 & $<0.05 \#$ \\
\hline Menisci extrusion change pre- to postop (mm \pm SD) & $1.50 \pm 1.9$ & $-0.94 \pm 1.9$ & $<0.05^{*}$ \\
\hline \multicolumn{4}{|l|}{ Radiographic imaging affected knee: } \\
\hline $\mathrm{KL}$ grade preop $0 / 1 / 2 / 3 / 4$ ( $n=2$ missing) & $6 / 9 / 1 / 0 / 0$ & $10 / 5 / 3 / 0 / 0$ & ns\# \\
\hline $\mathrm{KL}$ grade $\mathrm{FU} 0 / 1 / 2 / 3 / 4$ & $1 / 5 / 7 / 4 / 1$ & $7 / 4 / 4 / 3 / 0$ & ns\# \\
\hline
\end{tabular}

* Students t-test

${ }^{\#}$ Chi square test/Fisher's exact test

ns Non significant

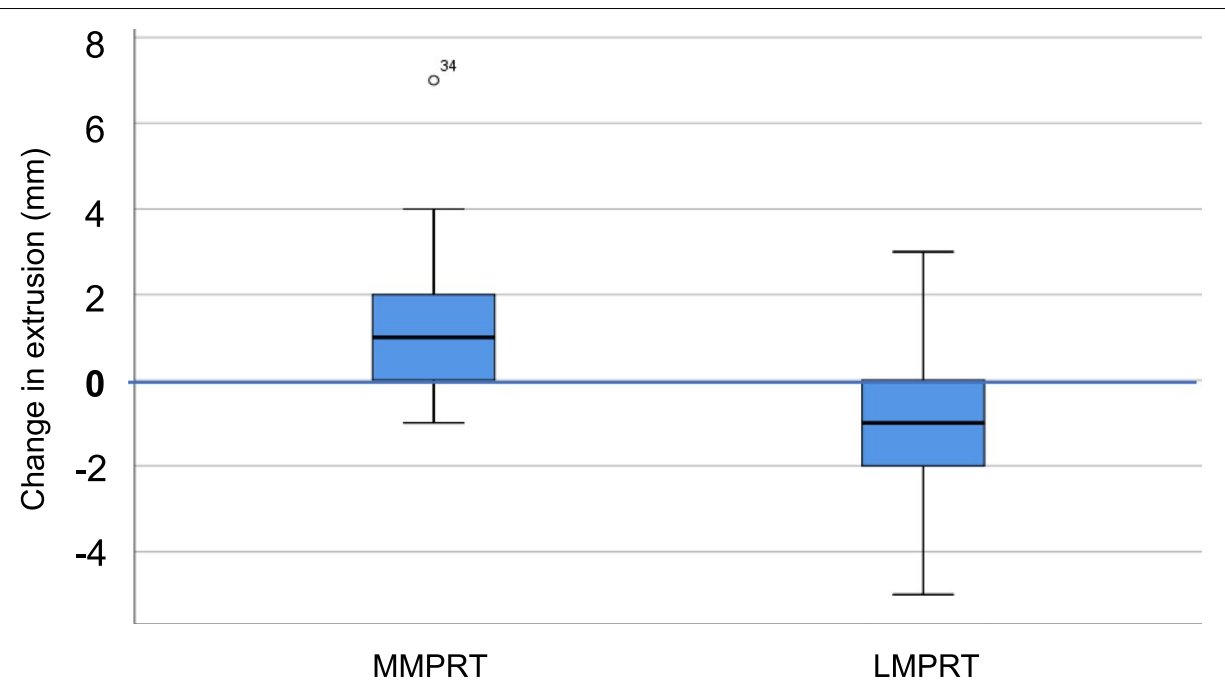

Fig. 3 Change in meniscal extrusion ( $\mathrm{mm}$ ) from pre-to postoperatively for the LMPRTs (lateral meniscus posterior root tears) and MMPRT (medial meniscus posterior root tears)

$(p<0.05)$ (Fig. 3). The inter-rater reliability (ICC) of the MRI scores was measured to be 0.58 (CI 0.15 to 1.0, SEM 0.19 ) for measurements on continuity and 0.74 (CI from 0.59 to 0.89 , SEM 0.06) for measurements on meniscal extrusion. (Table 3 ).

\section{Radiographic imaging}

Osteoarthritis according to the K-L classification of standard anteroposterior radiographs for the two groups are presented in Table 2. At follow-up the LMPRT group showed a mean change in K-L grade of 0.1 grade and the MMPRTs had a mean change of K-L grade from preoperatively to follow-up at 1.2 grades.
Table 3 Patient reported outcome (mean \pm standard deviation) for medial and lateral posterior meniscal root repairs at follow-up. (ADL, activity of daily living; QoL,quality of life)

\begin{tabular}{|c|c|c|c|}
\hline & $\begin{array}{l}\text { Medial root } \\
\text { repair }(n=18)\end{array}$ & $\begin{array}{l}\text { Lateral root } \\
\text { repair }(n=17)\end{array}$ & $P$-value* \\
\hline KOOS Symptoms ( \pm SD) & $76.6 \pm 16.9$ & $79.2 \pm 15.1$ & ns \\
\hline KOOS Pain ( \pm SD) & $70.5 \pm 26.1$ & $83.3 \pm 15.8$ & ns \\
\hline KOOS ADL ( $\pm S D)$ & $79.3 \pm 24.0$ & $91.9 \pm 11.1$ & ns \\
\hline KOOS Sports ( \pm SD) & $47.2 \pm 26.2$ & $59.7 \pm 17.1$ & ns \\
\hline KOOS QoL ( \pm SD) & $53.5 \pm 24.8$ & $60.7 \pm 23.7$ & ns \\
\hline Lysholm ( \pm SD) & $74.4 \pm 18.5$ & $79.2 \pm 16.0$ & ns \\
\hline Tegner ( \pm SD) & $2.3 \pm 2.1$ & $4.3 \pm 2.0$ & $<0.05$ \\
\hline GRoC Function ( \pm SD) & $4.1 \pm 1.2$ & $3.5 \pm 1.3$ & ns \\
\hline GRoC Pain ( \pm SD) & $4.2 \pm 1.3$ & $3.3 \pm 1.2$ & $<0.05$ \\
\hline
\end{tabular}




\section{Functional scores}

No differences between the two groups were found for all KOOS subscales and the Lysholm score. The Tegner activity scale was higher and the GRoC Pain score lower in the LMPRT group compared to the MMPRT group.

\section{Reoperations}

Four patients were reoperated due to complains related to the fixation button (1 MMPRT and 3 LMPRTs). Two MMPRTs were treated with high tibial osteotomy. One LMPRT patient had a partial meniscectomy and one was reoperated because of a cyclops lesion.

\section{Discussion}

The main finding of this study was that the majority of LMPRTs repaired by a transtibial suture technique showed continuity and healing of the meniscal fibres at the attachment site and the meniscal extrusion was diminished, whereas the MMPRTs had increased extrusion and showed less tendency of continuity of the fibres at attachment site according to MRI (28\% vs $61 \%)$. This study confirms that medial meniscus posterior root repairs are not able to restore anatomy back to normal state and the prevention of further degeneration may be postponed but not prevented by surgical intervention. Furthermore, this study confirmed that medial and lateral posterior meniscal root tears appear in different populations. Patients with LMPRTs were associated with traumatic injury and ACL reconstruction/revision reconstruction surgery. Patients with MMPRTs were associated with degeneration and progression of osteoarthritis and the patients were older and of higher BMI at operation.

The sustained extrusion of the medial menisci shown in this study, is in accordance with results from other studies: Chung et al. concluded that after repair, the meniscal extrusion was not reduced, and the technique did not prevent the development of OA [4]. In a systematic review of four different case-studies, Feucht et al. found that the healing was complete in 71 of 103 patients, partial in 29, and failed in 3. Meniscal extrusion however, decreased in only one of the four studies [8]. On the contrary, in LMPRTs, the outcome following repair has been found to be more promising: Okazaki et al. found a decrease in lateral meniscal extrusion following transtibial repair compared to other repair techniques and concluded with an increased chance to restore the hoop stresses in the menisci by this technique [29].

The diverging outcomes following MMPRTs compared to LMPRTs could have different explanations. First, most studies now reveal that there are demographic differences between the two groups. Krych et al. found that the LMPRTs were younger, had lower BMI, less cartilage degeneration and meniscal extrusion on MRI compared to patients with MMPRTs [20]. Secondly, patients with LMPRTs are often concomitantly treated with an ACL reconstruction. This could affect the outcome because the prospects of meniscal healing in general is improved when ACL reconstructions are performed in the same setting [19]. At last, lateral menisci with posterior root tears are less prone to excessive extrusion and the contact areas in the joint less diminished after LMPRT compared to MMPRT due to the meniscofemoral ligaments $[2,11]$. Therefore a reduction of extrusion could be easier to achieve on the lateral side.

Radiographic imaging showed a tendency towards progression of OA in the MMPRT group. To be able to conclude whether a repair might influence on OA ideally a control group, larger sample size and longer follow-up would have been of interest. Chung et al. found, in their metaanalysis on MMPRTs, that $35 \%$ of the patients with a MMPRT repair progressed according to the KL classification [4]. Pathological findings on MRI and radiographic imaging of the knee are not always correlated with clinical outcomes: Ulku et al. found a significant improvement in functional outcome after repair of MMPRTs even though a meniscal reduction was not achieved [36]. Other studies had similar findings for patients in the older age-group [5, 24].

The postoperative PROMs were similar in both groups, and in line with what has been reported in previously $[8,24]$. The reason for a lack of distinction between the LMPRTs and MMPRTs in the functional scores could be because many of the LMPRT patients were exposed to additional surgery, even ACL revision surgery ( 6 out of 18 patients) at the time of root repair. Previous studies have reported significant change in IKDC and Lysholm score after LMPRT and MMPRT repairs, ACL revisionreconstructions though, are known to result in less favorable clinical and functional outcomes than primary reconstructions [26].

The ICC scores for continuity were considered moderate and the ICC for meniscal extrusion was considered good. A standardized MRI scanner and a specified protocol should ideally have been performed to improve the reliability for the measurements.

Limitations to this study were the retrospective study design and a low number of subjects with variable follow-up of the patients. The osteoarthritis development is highly time dependent and the results could have been affected by this, although both study groups presented similar average follow-up period. Limb alignment and residual knee laxity should also have been addressed as they are important factors affecting the healingpotential. Meniscal root tears treated with non-operative treatments or other repair techniques were not evaluated 
in this study and no control group was available. However, previous studies have shown less favorable results in patients with non-operative treatment or with partial meniscectomy $[5,6,32]$. Other limitations were the lack of baseline PROMs. A baseline score of the study participants could ideally have given a more specific picture of the treatment effect. The learning curve of the surgeons is of concernwhen implementing a new technique, and this could possibly underestimate the treatment effect of the surgery. Finely, there is a lack of consensus on defining meniscal root healing on MRI, and a completely healed root repair was in this study only stated if continuity of the meniscus was confirmed in all three planes. Hence other studies might accept less strict criteria for healing compared to this study [20].

\section{Conclusion}

Following transtibial suture technique, repairs of LMPRTs had a higher tendency of healing compared to MMPRTs. Furthermore, repairs of MMPRTs showed progression of the meniscal extrusion despite surgical intervention. The study also confirmed that medial and lateral posterior meniscal root repairs appear in different patient groups.

\section{Authors' contributions}

The authors read and approved the final manuscript.

\section{Declarations}

\section{Competing interests}

The authors declare that they have no competing interest.

Received: 29 September 2021 Accepted: 17 November 2021 Published online: 09 December 2021

\section{References}

1. Allaire R, Muriuki M, Gilbertson L, Harner CD (2008) Biomechanical consequences of a tear of the posterior root of the medial meniscus. Similar to total meniscectomy. J Bone Joint Surg Am 90:1922-1931

2. Bao HR, Zhu D, Gong H, Gu GS (2013) The effect of complete radial lateral meniscus posterior root tear on the knee contact mechanics: a finite element analysis. J Orthop Sci 18:256-263

3. Bhatia S, LaPrade CM, Ellman MB, LaPrade RF (2014) Meniscal root tears: significance, diagnosis, and treatment. Am J Sports Med 42:3016-3030

4. Chung KS, Ha JK, Yeom CH, Ra HJ, Jang HS, Choi SH et al (2015) Comparison of clinical and radiologic results between partial Meniscectomy and Refixation of medial meniscus posterior root tears: a minimum 5-year follow-up. Arthroscopy 31:1941-1950

5. Dragoo JL, Konopka JA, Guzman RA, Segovia N, Kandil A, Pappas GP (2020) Outcomes of arthroscopic all-inside repair versus observation in older patients with meniscus root tears. Am J Sports Med 48:1127-1133

6. Faucett SC, Geisler BP, Chahla J, Krych AJ, Kurzweil PR, Garner AM et al (2019) Meniscus root repair vs Meniscectomy or nonoperative management to prevent knee osteoarthritis after medial meniscus root tears: clinical and economic effectiveness. Am J Sports Med 47:762-769
7. Feucht MJ, Izadpanah K, Lacheta L, Südkamp NP, Imhoff AB, Forkel P (2019) Arthroscopic transtibial pullout repair for posterior meniscus root tears. Oper Orthop Traumatol 31:248-260

8. Feucht MJ, Kühle J, Bode G, Mehl J, Schmal H, Südkamp NP et al (2015) Arthroscopic Transtibial pullout repair for posterior medial meniscus root tears: a systematic review of clinical, radiographic, and second-look arthroscopic results. Arthroscopy 31:1808-1816

9. Foreman SC, Liu Y, Nevitt MC, Neumann J, Joseph GB, Lane NE et al (2020) Meniscal root tears and extrusion are significantly associated with the development of accelerated knee osteoarthritis: data from the osteoarthritis initiative. Cartilage. https://doi.org/10.1177/194760352093452 51947603520934525

10. Forkel $P$, von Deimling $C$, Lacheta $L$, Imhoff FB, Foehr $P$, Willinger $L$ et al (2018) Repair of the lateral posterior meniscal root improves stability in an ACL-deficient knee. Knee Surg Sports Traumatol Arthrosc 26:2302-2309

11. Frank JM, Moatshe G, Brady AW, Dornan GJ, Coggins A, Muckenhirn KJ et al (2017) Lateral meniscus posterior root and Meniscofemoral ligaments as stabilizing structures in the ACL-deficient knee: a biomechanical study. Orthop J Sports Med 5:2325967117695756

12. Furumatsu T, Okazaki Y, Hiranaka T, Kodama Y, Kamatsuki Y, Zhang X et al (2020) An MRI-based suspension bridge sign can predict an arthroscopically favorable meniscal healing following the medial meniscus posterior root repair. J Orthop Sci. https://doi.org/10.1016/j.jos.2020.03.012

13. Hwang BY, Kim SJ, Lee SW, Lee HE, Lee CK, Hunter DJ et al (2012) Risk factors for medial meniscus posterior root tear. Am J Sports Med 40:1606-1610

14. Johnson DL, Swenson TM, Livesay GA, Aizawa H, Fu FH, Harner CD (1995) Insertion-site anatomy of the human menisci: gross, arthroscopic, and topographical anatomy as a basis for meniscal transplantation. Arthroscopy 11:386-394

15. Kamper SJ, Maher CG, Mackay G (2009) Global rating of change scales: a review of strengths and weaknesses and considerations for design. J Man Manip Ther 17:163-170

16. Kellgren JH, Lawrence JS (1957) Radiological assessment of osteo-arthrosis. Ann Rheum Dis 16:494-502

17. Kim JH, Chung JH, Lee DH, Lee YS, Kim JR, Ryu KJ (2011) Arthroscopic suture anchor repair versus pullout suture repair in posterior root tear of the medial meniscus: a prospective comparison study. Arthroscopy 27:1644-1653

18. Kim SB, Ha JK, Lee SW, Kim DW, Shim JC, Kim JG et al (2011) Medial meniscus root tear refixation: comparison of clinical, radiologic, and arthroscopic findings with medial meniscectomy. Arthroscopy 27:346-354

19. Korpershoek JV, de Windt TS, Vonk LA, Krych AJ, Saris DBF (2020) Does anterior cruciate ligament reconstruction protect the meniscus and its repair? A systematic review. Orthop J Sports Med 8:2325967120933895

20. Krych AJ, Bernard CD, Kennedy NI, Tagliero AJ, Camp CL, Levy BA et al (2020) Medial versus lateral meniscus root tears: is there a difference in injury presentation, treatment decisions, and surgical repair outcomes? Arthroscopy 36:1135-1141

21. LaPrade CM, Foad A, Smith SD, Turnbull TL, Dornan GJ, Engebretsen L et al (2015) Biomechanical consequences of a nonanatomic posterior medial meniscal root repair. Am J Sports Med 43:912-920

22. LaPrade CM, James EW, Cram TR, Feagin JA, Engebretsen L, LaPrade RF (2015) Meniscal root tears: a classification system based on tear morphology. Am J Sports Med 43:363-369

23. LaPrade CM, Jansson KS, Dornan G, Smith SD, Wijdicks CA, LaPrade RF (2014) Altered tibiofemoral contact mechanics due to lateral meniscus posterior horn root avulsions and radial tears can be restored with in situ pull-out suture repairs. J Bone Joint Surg Am 96:471-479

24. LaPrade RF, Matheny LM, Moulton SG, James EW, Dean CS (2017) Posterior meniscal root repairs: outcomes of an anatomic Transtibial pull-out technique. Am J Sports Med 45:884-891

25. Lerer DB, Umans HR, Hu MX, Jones MH (2004) The role of meniscal root pathology and radial meniscal tear in medial meniscal extrusion. Skelet Radiol 33:569-574

26. Lind M, Menhert F, Pedersen AB (2012) Incidence and outcome after revision anterior cruciate ligament reconstruction: results from the Danish registry for knee ligament reconstructions. Am J Sports Med 40:1551-1557 
27. Magee T (2008) MR findings of meniscal extrusion correlated with arthroscopy. J Magn Reson Imaging 28:466-470

28. Nguyen JC, De Smet AA, Graf BK, Rosas HG (2014) MR imaging-based diagnosis and classification of meniscal tears. Radiographics 34:981-999

29. Okazaki Y, Furumatsu T, Kamatsuki Y, Masuda S, Hiranaka T, Kodama Y et al (2020) Transtibial pullout repair of the lateral meniscus posterior root tear combined with anterior cruciate ligament reconstruction reduces lateral meniscus extrusion: a retrospective study. Orthop Traumatol Surg Res 106:469-473

30. Padalecki JR, Jansson KS, Smith SD, Dornan GJ, Pierce CM, Wijdicks CA et al (2014) Biomechanical consequences of a complete radial tear adjacent to the medial meniscus posterior root attachment site: in situ pull-out repair restores derangement of joint mechanics. Am J Sports Med 42:699-707

31. Palisch AR, Winters RR, Willis MH, Bray CD, Shybut TB (2016) Posterior root meniscal tears: preoperative, intraoperative, and postoperative imaging for Transtibial pullout repair. Radiographics 36:1792-1806

32. Pareek A, Parkes CW, Bernard C, Camp CL, Saris DBF, Stuart MJ et al (2020) Spontaneous osteonecrosis/Subchondral insufficiency fractures of the knee: high rates of conversion to surgical treatment and Arthroplasty. J Bone Joint Surg Am 102:821-829

33. Ren AH, Zheng ZZ, Shang Y, Tian CY (2012) An anatomical study of normal meniscal roots with isotropic 3D MRI at 3T. Eur J Radiol 81:e783-e788

34. Roos EM, Roos HP, Lohmander LS, Ekdahl C, Beynnon BD (1998) Knee injury and osteoarthritis outcome score (KOOS)--development of a selfadministered outcome measure. J Orthop Sports Phys Ther 28:88-96

35. Tegner Y, Lysholm J (1985) Rating systems in the evaluation of knee ligament injuries. Clin Orthop Relat Res. 1985;198:43-49

36. Ulku TK, Kaya A, Kocaoglu B (2020) Suture configuration techniques have no effect on mid-term clinical outcomes of arthroscopic meniscus root repairs. Knee 27:676-682

\section{Publisher's Note}

Springer Nature remains neutral with regard to jurisdictional claims in published maps and institutional affiliations.

\section{Submit your manuscript to a SpringerOpen ${ }^{\circ}$ journal and benefit from:}

- Convenient online submission

- Rigorous peer review

- Open access: articles freely available online

- High visibility within the field

- Retaining the copyright to your article

Submit your next manuscript at $\gg$ springeropen.com 